\title{
NIBIT-MESO-1: limitations and clinical perspectives in MPM treatment testing an immune checkpoint blockade combination in a single-arm study
}

\author{
Paolo Bironzo ${ }^{1}$, Riccardo Taulli ${ }^{2}$ \\ ${ }^{1}$ Thoracic Unit and Medical Oncology Division, Department of Oncology at San Luigi Hospital, University of Torino, Orbassano, Italy; \\ ${ }^{2}$ Department of Oncology, University of Torino, Torino, Italy \\ Correspondence to: Riccardo Taulli. Department of Oncology, University of Torino, Via Santena 5, 10126, Torino, Italy. Email: riccardo.taulli@unito.it. \\ Provenance: This is an invited Editorial commissioned by the Section Editor Zhenying Guo (Department of Pathology, Zhejiang Cancer Hospital, \\ Hangzhou, China). \\ Comment on: Calabrò L, Morra A, Giannarelli D, et al. Tremelimumab combined with durvalumab in patients with mesothelioma (NIBIT-MESO-1): \\ an open-label, non-randomised, phase 2 study. Lancet Respir Med 2018;6:451-60.
}

Submitted Aug 03, 2018. Accepted for publication Sep 27, 2018.

doi: $10.21037 /$ jtd.2018.09.135

View this article at: http://dx.doi.org/10.21037/jtd.2018.09.135

Malignant pleural mesothelioma (MPM), which is linked to asbestos exposure and is a rare, lethal disease of the serosal membrane in the chest, has an incidence which continues to increase worldwide $(1,2)$. MPM is histologically classified into three major subtypes: epithelioid, biphasic (or mixed) and sarcomatoid. Notwithstanding these well-defined MPM histological subtypes, there are tremendous differences in clinical outcomes including long-term survival in patients whose tumor histology is indistinguishable from conventional, highly aggressive MPM.

MPM is generally diagnosed in the late stage where chemotherapy remains the only therapeutic option. Unfortunately, the efficacy of chemotherapy is modest, with a median overall survival of about 12 months (3). This limited efficacy has been associated with MPM's ability to evade the immune system clearance and to establish an immune-suppressive environment $(4,5)$. Consequently, it is not surprising that patients enrolled in the MAPS phase 3 trial and in the LUME-Meso phase 2 trial benefited from the addition of bevacizumab and nintedanib to the standard cisplatin and pemetrexed backbone, respectively. In a small phase $1 \mathrm{~b}$ non-randomized open-label study, the treatment with the anti-PD-1 monoclonal antibody pembrolizumab, was well tolerated, leading to a $20 \%$ response rate (RR) and a median duration of response of 12 months (95\% CI: 6.8-40.7) in previously treated MPM patients (6). A phase 2 study of the same agent in previously treated
MPM patients, unselected for PD-L1 expression, led to comparable results, showing an overall RR (ORR) of $22 \%$ and a disease-control rate (DCR) of $61 \%$ (7). Interestingly, the objective response was correlated to the PD-L1 expression. Moreover, data with nivolumab in $27 \mathrm{MPM}$ patients treated in the American Nivolumab Expanded Access Program showed an ORR of $24 \%$ while the DCR and the 6 -month survival rate were $60 \%$ and $52 \%$, respectively (8). Similarly, phase 1 data on avelumab, which is another checkpoint inhibitor directed against PD-L1, demonstrated a lower RR (9.4\%) but comparable median OS (10.9 months), which was even higher in $\mathrm{PD}$-L1 positive cases $(\geq 5 \%$ assessed by DAKO 73-10 antibody, 22.0 months) (9).

To improve the efficacy of immune modulators and to counteract phenomena of resistance, the combination of immune checkpoint blocking antibodies has been investigated in other tumor types. In metastatic melanoma, for instance, the association of nivolumab with ipilimumab, whose combined use has been approved by the US Food and Drug Administration, has improved the overall survival (10). This pivotal clinical study has shed light on the fundamentals for using combinations of immune checkpoint blocking antibodies in different tumor types (11). In line with this, an NIBIT-MESO-1 trial (12), has recently investigated the activity of tremelimumab (anti-CTLA-4 monoclonal antibody) in combination with durvalumab (anti-PD-L1 monoclonal antibody), in first- or second- 
line treatment for MPM patients. The clinical activity of CTLA-4 blockade in monotherapy was previously reported in second-line treatment for MPM patients, although the efficacy was not confirmed in the randomized, placebocontrolled phase 2 DETERMINE study $(13,14)$.

NIBIT-MESO-1 (12) is a single-arm, single-centre, open-label phase 2 study which included adult patients with histologically confirmed, unresectable pleural or peritoneal MM. Patient inclusions encompassed cases that had refused first-line chemotherapy, cases with disease progression during or after standard chemotherapy treatment, and patients with lesions measurable and suitable for repeated assessments according to standard evaluation criteria for both pleural and peritoneal MM. Patients excluded were those previously treated with monoclonal antibodies against CTLA-4, PD-1, or PD-L1 or those with active immune diseases or brain metastases. The pharmacokinetic was based on a previous, phase $1 \mathrm{~b}$ trial in non-small-cell lung cancer. Specifically, the induction phase consisted of 4 cycles of tremelimumab (at the dosage of $1 \mathrm{mg} / \mathrm{kg}$ ) in association with durvalumab (at the dosage of $20 \mathrm{mg} / \mathrm{kg}$ ), both administered intravenously every 4 weeks. The maintenance phase consisted of durvalumab at the same dosage and schedules as in the induction, but administered for up to 9 cycles. Follow-up is still ongoing. The primary endpoint was the proportion of patients with an immunerelated objective response (complete response or partial response) according to immune-related criteria for patients with pleural and peritoneal mesothelioma. Secondary endpoints included safety, proportion of patients with immune-related disease control (complete response, partial response, or stable disease), immune-related progressionfree survival, progression-free survival, and overall survival. The association between individual efficacy endpoints and baseline PD-L1 levels was also included among the secondary endpoints. Safety was assessed monthly after each dose administration both during induction and maintenance phases. After the last administration of tremelimumab and durvalumab, safety was assessed every 30 days for up to 90 days, while patients with severe adverse effects were monitored for one year.

Since fewer than $30 \%$ of all treatment-related adverse events (grade 3-4) were observed in the first 10 patients, all the originally planned patients (40 in total) were enrolled. Immune-related objective response was observed in 11 patients and confirmed in 10 patients by CT scan. The median duration of immune-related partial response in these patients was 16.1 months and thus the study reached its primary endpoint. Immune-related disease control, with a median duration of 10.6 months, was observed in 26 patients, while the median immune-related progression-free survival was 8 months, and immune-related progressionfree survival was $55 \%$ at 6 months and $28 \%$ at 12 months. Median progression-free survival and median overall survival were 5.7 and 16.6 months, respectively, with 25 patients still being alive at 1 year. Sixty-eight percent of patients experienced grade 1 or 2 treatment-related adverse events (most dermatological), while grade 3 and 4 occurred in $18 \%$ of patients. No treatment-related deaths were reported. Overall, this study describes a tolerable safety profile for the combination and hints at its potentially clinical benefit.

A recent French randomized phase 2 non-comparative study assessed the efficacy of nivolumab alone or in combination with ipilimumab in pre-treated advanced MPM patients. In the 62 patients treated in the combination arm, the median PFS was 5.6 and 4.0 months for those treated with nivolumab alone $(\mathrm{n}=63)$ (15). Although data are still not mature, at a median follow-up of 15 months, median OS was 13.6 months in the single-agent arm, while it was not even reached with the combination of drugs.

Unfortunately, it is difficult make comparisons between the above studies due to the profound differences in the trial design, the enrolled populations (e.g., histological subtypes, percentage of peritoneal mesotheliomas, adopted response evaluation criteria) and the agents used (single-agent $v s$. combination therapies). However, in spite of such intrinsic limitations, the median progression-free survival and the median overall survival of the NIBIT-MESO-1 trial were comparable with those observed in the KEYNOTE-028 study, in which patients were selected according to PD-L1 levels. Notably, in the NIBIT-MESO-1 trial, the level of PD-L1 at the baseline was not significantly associated to any clinical response (immune-related objective response, immune-related disease control, immune-related progression-free survival or 1-year overall survival). This observation is particularly relevant, since in many studies with immune modulators, the level of PD-L1 is still considered a relevant parameter for effectiveness $(16,17)$. However, the findings here reported suggest that additional criteria are urgently needed. A detailed molecular analysis at the genomic and transcriptional level of the tumor and the relative microenvironment, could be extremely informative in identifying novel critical biomarkers to improve patient stratification and selection. In other tumor types, the mutational burden (TMB) seems 
to be a useful parameter in identifying good responders to immune modulators (18).

Compared to other malignancies, MPM is characterized by fewer recurrent genetic alterations (19). At the same time, MPM is reported to be associated with very low TMB, as recently reported by Ladanyi and colleagues who analyzed The Cancer Genome Atlas (20). Yet, in the same study, the authors found that another negative immune checkpoint, VISTA, was highly expressed in this tumor as compared to the others, a fact which begs further research from future studies.

Unfortunately, these single-arm studies suggest hints of anti-cancer activity but do not allow us to draw any decisive conclusion in the absence of an internal comparative arm. Although there are no officially accepted second lines in MPM, non-approved agents such as gemcitabine vinorelbine are commonly used in this setting.

Overall, the NIBIT-MESO-1 study (12), despite its limitations, suggests that the combination of tremelimumab and durvalumab has an acceptable tolerability and leads to some clinical benefit in MM patients. The trade-offs between activity and toxicity do still need to be carefully assessed, and consequently, further well-designed studies are needed to extend and consolidate these findings. As pointed out by Calabrò et al., within this innovative context of combination therapies, the use of two-immune checkpoint blocking antibodies in association with other drugs might further improve clinical benefits in the near future. While ongoing clinical trials are mainly focusing on adding immune checkpoint inhibitors to chemotherapy in the first-line treatment, early preclinical data indicate that bromodomain inhibitors (BBIs) can be used as effective agents in restoring an immune-active environment in MPM (21). Nevertheless, caution should be applied before combining many drugs in a clinical trial, since the resulting toxicity may be intolerably exponential.

\section{Acknowledgements}

$\mathrm{R}$ Taulli research is supported by AIRC.

\section{Footnote}

Conflicts of Interest: The authors have no conflicts of interest to declare.

\section{References}

1. Tweedale G. Asbestos and its lethal legacy. Nat Rev Cancer
2002;2:311-5.

2. Robinson BWS, Lake RA. Advances in malignant mesothelioma. N Engl J Med 2005;353:1591-603.

3. Remon J, Lianes P, Martínez S, et al. Malignant mesothelioma: new insights into a rare disease. Cancer Treat Rev 2013;39:584-91.

4. Izzi V, Masuelli L, Tresoldi I, et al. Immunity and malignant mesothelioma: from mesothelial cell damage to tumor development and immune response-based therapies. Cancer Lett 2012;322:18-34.

5. Aerts JG, Lievense LA, Hoogsteden HC, et al. Immunotherapy prospects in the treatment of lung cancer and mesothelioma. Transl Lung Cancer Res 2014;3:34-45.

6. Alley EW, Lopez J, Santoro A, et al. Clinical safety and activity of pembrolizumab in patients with malignant pleural mesothelioma (KEYNOTE-028): preliminary results from a non-randomised, open-label, phase $1 \mathrm{~b}$ trial. Lancet Oncol 2017;18:623-30.

7. Desai A, Karrison T, Rose B, et al. Phase II trial of pembrolizumab $(\mathrm{P})$ in patients (pts) with previously-treated mesothelioma (MM). J Clin Oncol 2018;36:abstr 8565.

8. Hamad H, Shafi S, Lenge De Rosen V, et al. A realworld experience of nivolumab in advanced malignant mesothelioma (MM). J Clin Oncol 2018;36:abstr 8569.

9. Hassan R, Thomas A, Nemunaitis JJ, et al. Phase 1b study of avelumab in advanced previously treated mesothelioma: long-term follow-up from JAVELIN solid tumor. J Clin Oncol 2018;36:abstr 8563.

10. Larkin J, Chiarion-Sileni V, Gonzalez R, et al. Combined Nivolumab and Ipilimumab or Monotherapy in Untreated Melanoma. N Engl J Med 2015;373:23-34.

11. Zappasodi R, Merghoub T, Wolchok JD. Emerging Concepts for Immune Checkpoint Blockade-Based Combination Therapies. Cancer Cell 2018;33:581-98.

12. Calabrò L, Morra A, Giannarelli D, et al. Tremelimumab combined with durvalumab in patients with mesothelioma (NIBIT-MESO-1): an open-label, non-randomised, phase 2 study. Lancet Respir Med 2018;6:451-60.

13. Calabrò L, Morra A, Fonsatti E, et al. Tremelimumab for patients with chemotherapy-resistant advanced malignant mesothelioma: an open-label, single-arm, phase 2 trial. Lancet Oncol 2013;14:1104-11.

14. Maio M, Scherpereel A, Calabrò L, et al. Tremelimumab as second-line or third-line treatment in relapsed malignant mesothelioma (DETERMINE): a multicentre, international, randomised, double-blind, placebocontrolled phase 2b trial. Lancet Oncol 2017;18:1261-73. 15. Zalcman G, Mazieres J, Greillier L, et al. LBA58_PR 
Second or 3rd line nivolumab (Nivo) versus nivo plus ipilimumab (Ipi) in malignant pleural mesothelioma (MPM) patients: Updated results of the IFCT-1501 MAPS2 randomized phase 2 trial. Ann Oncol 2017;28.

16. Manson G, Norwood J, Marabelle A, et al. Biomarkers associated with checkpoint inhibitors. Ann Oncol 2016;27:1199-206.

17. Garon EB, Rizvi NA, Hui R, et al. Pembrolizumab for the treatment of non-small-cell lung cancer. N Engl J Med 2015;372:2018-28.

18. Schumacher TN, Schreiber RD. Neoantigens in cancer immunotherapy. Science 2015;348:69-74.

19. Bueno R, Stawiski EW, Goldstein LD, et al. Comprehensive genomic analysis of malignant pleural

Cite this article as: Bironzo P, Taulli R. NIBIT-MESO-1: limitations and clinical perspectives in MPM treatment testing an immune checkpoint blockade combination in a singlearm study. J Thorac Dis 2018;10(Suppl 33):S3878-S3881. doi: $10.21037 / \mathrm{jtd} .2018 .09 .135$ mesothelioma identifies recurrent mutations, gene fusions and splicing alterations. Nat Genet 2016;48:407-16.

20. Ladanyi M, Robinson BW, Campbell PJ. The TCGA malignant pleural mesothelioma (MPM) project: VISTA expression and delineation of a novel clinical-molecular subtype of MPM. J Clin Oncol 2018;36:abstr 8516.

21. Riganti C, Lingua MF, Salaroglio IC, et al. Bromodomain inhibition exerts its therapeutic potential in malignant pleural mesothelioma by promoting immunogenic cell death and changing the tumor immune-environment. Oncoimmunology 2017;7:e1398874.

(English Language Editor: John Gray, AME Publishing Company) 